\title{
HYDROXYL RADICAL PRODUCTION IN DC CORONA STREAMER DISCHARGE
}

\author{
Yiyi Zhao, Mark P. Wilson, Tao Wang, Igor V. Timoshkin, Scott J. MacGregor \\ Electronic and Electrical Engineering, University of Strathclyde \\ Glasgow, G1 1XW, United Kingdom \\ y.zhao@strath.ac.uk
}

\begin{abstract}
Plasma-induced advanced oxidation processes do not suffer from the drawbacks, such as carcinogenic byproducts, associated with conventional water treatment, and enable the removal of micro-pollutants. The high oxidation strength of hydroxyl radicals enables degradation of resistant contaminants. Many reactions are known to occur at the plasma-water interface; however, the mechanisms of hydroxyl radical production are still not clear.

To understand the physical and chemical processes occurring at the plasma-water interface, this research involved investigation of the hydroxyl radicals produced during d.c. streamer discharges. A needleplate electrode configuration in atmospheric air was used, with the treated solution used as the ground electrode. To understand the effects of polarity and gas type on hydroxyl radical production, both positive- and negative-polarity energization in air, nitrogen and helium were investigated. Plasma filaments were developed from the needle electrode, which was in contact with the solution. Terephthalic acid (TA) was used as a scavenger of hydroxyl $(\mathrm{OH})$ radicals, with $\mathrm{OH}$ density subsequently being quantified by fluorescence emission from 2-hydroxyterephthalic acid (HTA), which is formed through specific reaction between TA and $\mathrm{OH}$.
\end{abstract}

The power inputs in positive pulsed streamer discharges were $0.125 \mathrm{~W}, 0.18 \mathrm{~W}$ and $0.26 \mathrm{~W}$ in air, nitrogen and helium, respectively; the corresponding hydroxyl radical production efficiencies were $0.56 \mathrm{mmol} / \mathrm{kWh}, 1.1 \mathrm{mmol} / \mathrm{kWh}$ and $5.94 \mathrm{mmol} / \mathrm{kWh}$, respectively. For negative pulsed streamer discharges in air, the power input was $0.063 \mathrm{~W}$ and the efficiency was $1 \mathrm{mmol} / \mathrm{kWh}$. The hydroxyl radical production rates were $2.6 \times 10^{-7} \mathrm{Ms}^{-1}$ in negative air discharges, and $2.7 \times 10^{-7} \mathrm{Ms}^{-1}, 1.8 \times 10^{-6} \mathrm{Ms}^{-1}$, and $2.2 \times$ $10^{-6} \mathrm{Ms}^{-1}$ in positive air, nitrogen and helium discharges, respectively.

\section{INTRODUCTION}

Advanced oxidation processes (AOPs) using $\mathrm{O}_{3}$, $\mathrm{H}_{2} \mathrm{O}_{2}$, UV or Fenton reactions have shown potential in treating hard-degradation wastewater. Hydroxyl radicals produced in the process possess high oxidation ability and the reactions do not produce carcinogenic by-products [3]; they can react with substances in a non-selective manner, and the general reaction constant is more than $10^{9} \mathrm{Lmol}^{-1} \mathrm{~s}^{-1}$ [4]. As an alternative to the traditional AOP process, research in non-thermal plasma induced AOPs has been extensively investigated in the past decades. The reactions at the plasma-water interface lead to chemical activations by producing active species in-situ, including ions, reactive radicals, excited molecules and atoms, without the requirement for additional chemicals [5].

Different types of gas discharge have been investigated for the oxidation process including corona, glow, arc and dielectric barrier discharge. Kanazawa found hydroxyl radical production rate of the order of $10^{-9} \mathrm{Ms}^{-1}$ by surface pulsed streamer discharge [6] and of the order of $10^{-8} \mathrm{Ms}^{-1}$ by plasma jet treatment [7]. $\mathrm{Li}$ et al. [8] have estimated an $\mathrm{OH}$ production rate of the order of $10^{-10} \mathrm{Ms}^{-1}$ by pulsed discharges in water, measured using salicylic acid.

This research quantifies the hydroxyl radical production rate in different gases at the plasma-water interface, by measuring the density of hydroxyl radicals in the plasma. A pin-to-plate configured discharge reactor was developed to investigate the interfacial reactions and production efficiency.

\section{EXPERIMENTAL SET UP}

\section{A. Reactor Design}

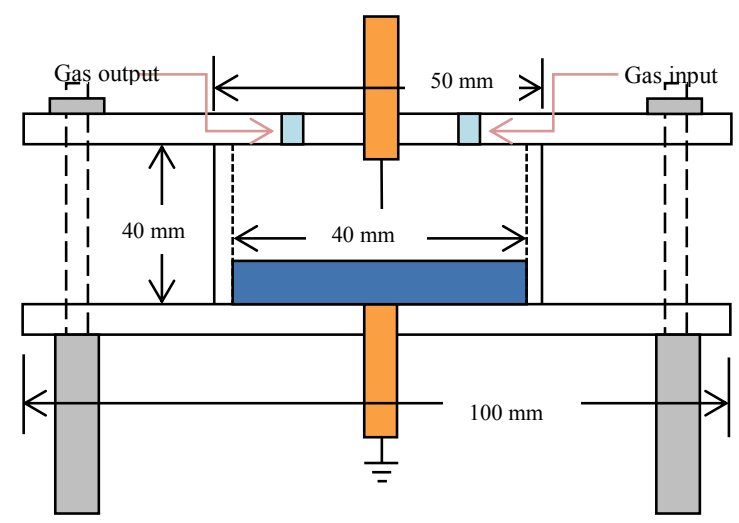

Figure 1. Streamer discharge plasma reactor.

The streamer discharge reactor is shown in Fig. 1. The reactor consists of a typical pin-to-plate 
configuration. The high-voltage electrode consists of a steel needle with a tip diameter of $0.3 \mathrm{~mm}$, protruding from a copper rod, and fixed at a distance of $1 \mathrm{~mm}$ above the water surface. A grounded copper rod electrode is positioned centrally in the lower flange, which is made of nylon. The total sample volume was $10 \mathrm{ml}$, introduced into the reactor by a $5 \mathrm{ml}$ pipette (P5000G, Gilson).

Since various feed gases (air, nitrogen and helium) were used, a reactor vacuuming process was followed between tests with different gases. The discharge chamber was made of PTFE, with upper and lower nylon discs acting as flanges to seal the chamber during vacuuming. The gas inlet and outlet ports were located on the upper nylon disc.

\section{B. Experimental Set-up}

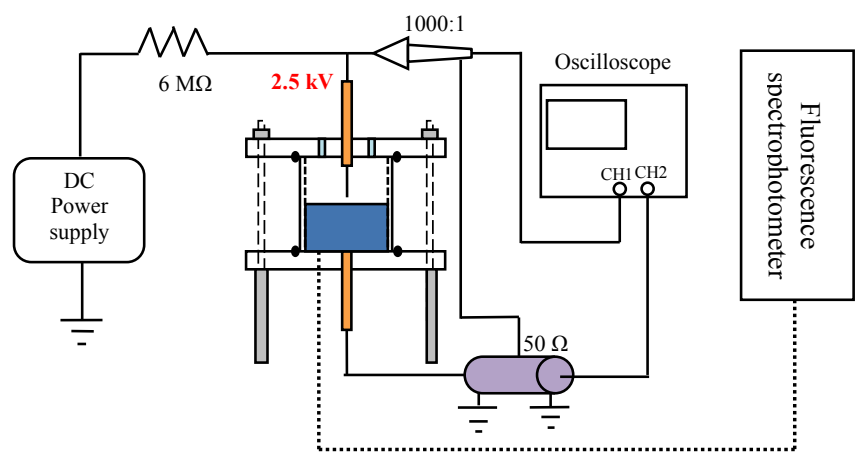

Figure 2. Experimental set-up.

The experimental set-up is shown in Fig. 2. A d.c. power supply $(0-20 \mathrm{kV})$ with changeable polarity was utilized to provide a discharge voltage of $2.5 \mathrm{kV}$. A 6 $\mathrm{M} \Omega$ protection resistor was connected in series with the reactor, which minimises the d.c. component of the pulsed discharge current. A Tektronix P6015A highvoltage probe (bandwidth $75 \mathrm{MHz}$ ) was employed to measure the voltage applied to the reactor. A $50 \Omega$ coaxial cable was connected to the grounded electrode of the reactor to measure the current waveform. A Teledyne Lecroy digital oscilloscope (WaveRunner 6 $\mathrm{Zi)}$ with a bandwidth of $4 \mathrm{GHz}$ and sampling rate of $40 \mathrm{GS} / \mathrm{s}$ was used to record waveform data. A fluorescence spectrophotometer (RF5301PC, Shimadzu Scientific Instruments) was employed to measure the concentration of 2-hydroxyterephthalic acid, the production of hydroxyl radical with terephthalic acid, to determine the concentration of hydroxyl radicals produced.

\section{Sample Preparation and Treatment}

$200 \mathrm{mg}$ sodium hydroxide (ACS reagent, $\geq 97.0 \%$, pellets Sigma-Aldrich) was weighed and dissolved in $1000 \mathrm{ml}$ water to make $5 \mathrm{mM}$ sodium hydroxide solution. $332 \mathrm{mg}$ terephthalic acid (98\%, Aldrich) was weighed and dissolved in $1000 \mathrm{ml} 5 \mathrm{mM}$ sodium hydroxide solution, standing the solution for two hours to completely dissolve the terephthalic acid. 2hydroxyterephthalic acid (97\%, Aldrich) was used to make up and plot the calibration curve (following the method in [2]) to determine the amount of hydroxyl radicals, which, in turn, was used to calculate production rate.

Experiments were conducted with treatment times of 5,10 , and 15 minutes, in air, nitrogen and helium under positive polarity; and in air under negative polarity. Each treatment was repeated 3 times.

\section{RESULTS AND DISCUSSION}

\section{A. Different Gas Effect under Positive Polarity \\ 1) Voltage and Current Comparison}

Different feed gas results in entirely different voltage and current waveforms. The onset of the voltage drop in helium is quick, the voltage starting to fall as soon as the discharge occurs, and the voltage decreases at a much faster rate than in air and nitrogen. The onset of the voltage drop in air and nitrogen is gradual, and the voltage falls at a much slower rate compared with helium. The discharge current in helium is of shorter duration $(1 \mu \mathrm{s})$ when compared to that in air and nitrogen $(2 \mu \mathrm{s})$, and of higher amplitude $(\sim 750 \mathrm{~mA})$, when compared to that in air $(\sim 50 \mathrm{~mA})$ and nitrogen ( $>80 \mathrm{~mA})$.
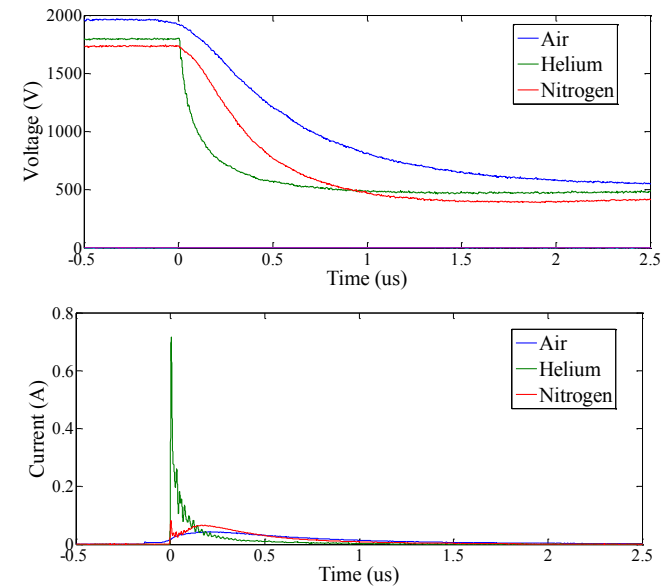

Figure 3. Effect of gas type on voltage and current waveforms.

During a positive streamer discharge, positive ions gather at the streamer head and move towards ground, electrons gathering at the streamer tail and moving in the opposite direction, towards the high-voltage electrode. The streamer body consists of a mixture of positive ions and electrons, these charges moving slower than those at the head and tail, and arriving at the ground and high-voltage electrodes later. In all gas types, the current pulse has a short-duration front, followed by a long-duration tail, but the tail duration in helium is shorter than in air and nitrogen, which means that the time for positive ions in air and nitrogen streamer bodies to arrive at the water surface is longer than that for positive ions in helium streamers.

\section{2) Power and Charge Transfer Comparison}


Table 1 show the power and charge transfer in different gases for positive discharges. The discharge frequency in air is $2 \mathrm{kHz}$, which is lower than that in nitrogen and helium, at $5 \mathrm{kHz}$. For positive discharges in different gases, air discharge has the lowest power and charge transfer per second, due to the low discharge frequency. The power and charge transfer in nitrogen is $44 \%$ and $48 \%$ higher than air, respectively; while helium has the highest power input and charge transfer, being $108 \%$ and $80 \%$ higher than air, respectively.

Table 1. Power and charge transfer for positive discharges in different gases.

\begin{tabular}{|c|c|c|c|}
\hline Gas & $\begin{array}{c}\text { Power } \\
(\mathrm{W})\end{array}$ & $\begin{array}{c}\text { Charge } \\
\text { transfer/pulse } \\
(\mathrm{nC})\end{array}$ & $\begin{array}{c}\text { Charge } \\
\text { Transfer/s } \\
(\mu \mathrm{C})\end{array}$ \\
\hline Air & 0.125 & 57.5 & 115 \\
\hline Nitrogen & 0.18 & 34.2 & 171 \\
\hline Helium & 0.26 & 41.8 & 209 \\
\hline
\end{tabular}

\section{3) Hydroxyl Radical Production}

For positive streamer discharges, the gas type influences hydroxyl radical production. For all gas types, the hydroxyl radical concentration increased linearly with the treatment time up to 15 minutes. For 15 minutes treatment, the hydroxyl radical production rate was $2.7 \times 10^{-7} \mathrm{Ms}^{-1}$ in air, $1.8 \times 10^{-6} \mathrm{Ms}^{-1}$ in nitrogen and $2.2 \times 10^{-6} \mathrm{Ms}^{-1}$ in helium, respectively. The number of hydroxyl radicals produced using helium is 6.5 times higher than using air. While the effect is less pronounced using nitrogen rather than helium, the number of hydroxyl radicals produced is still 4.5 times higher than using air. In positive streamer discharges, ions are considered as the main contribution to hydroxyl radical production.

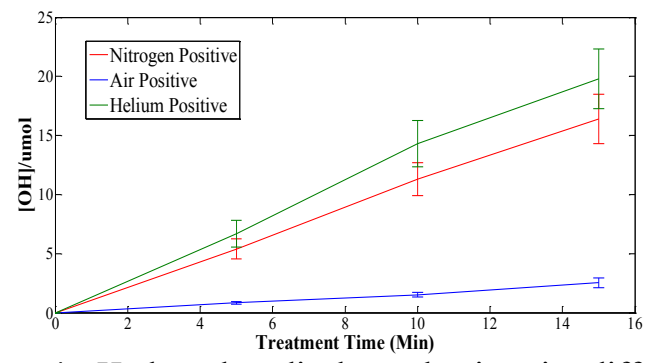

Figure 4. Hydroxyl radical production in different gases.

When energetic electrons collide with oxygen, nitrogen and helium gas molecules, one of the outermost electrons will be removed or the orbit changed, thus, the gas molecules become positive ions $\left(\mathrm{N}_{2}{ }^{+}, \mathrm{He}^{+}, \mathrm{O}_{2}{ }^{+}\right)$, or excited-state molecules. The positive ions and exited-state molecule can further react with water to form hydroxyl radicals and ground-state gas molecules. The chemical reactions in the gases used in this study are summarized below (Eq. (1) to Eq. (10)):

Nitrogen reactions:

$$
\begin{gathered}
\mathrm{N}_{2}+\mathrm{e} \rightarrow \mathrm{N}_{2}^{+}+2 \mathrm{e}(\mathrm{E}=14.6 \mathrm{eV}) \\
\mathrm{N}_{2}^{+}+\mathrm{H}_{2} \mathrm{O} \rightarrow \mathrm{N}_{2}+\mathrm{H}_{2} \mathrm{O}^{+}
\end{gathered}
$$

Helium reactions:

$$
\begin{aligned}
& \mathrm{H}_{2} \mathrm{O}^{+}+\mathrm{H}_{2} \mathrm{O} \rightarrow \mathrm{H}_{3} \mathrm{O}^{+}+\mathrm{OH} \\
& \mathrm{N}_{2}{ }^{+}+\mathrm{H}_{2} \mathrm{O} \rightarrow \mathrm{OH}+\mathrm{H}+\mathrm{N}_{2}
\end{aligned}
$$

$$
\begin{gathered}
\mathrm{He}+\mathrm{e} \rightarrow \mathrm{He}^{+}+2 \mathrm{e}(\mathrm{E}=24.6 \mathrm{eV}) \\
\mathrm{He}^{+}+\mathrm{H}_{2} \mathrm{O} \rightarrow \mathrm{He}+\mathrm{H}_{2} \mathrm{O}^{+} \\
\mathrm{H}_{2} \mathrm{O}^{+}+\mathrm{H}_{2} \mathrm{O} \rightarrow \mathrm{H}_{3} \mathrm{O}^{+}+\mathrm{OH}
\end{gathered}
$$

Oxygen reactions:

$$
\begin{gathered}
\mathrm{O}_{2}+\mathrm{e} \rightarrow \mathrm{O}_{2}^{+}+2 \mathrm{e}(\mathrm{E}=13.6 \mathrm{eV}) \\
\mathrm{O}_{2}^{+}+\mathrm{H}_{2} \mathrm{O} \rightarrow \mathrm{O}_{2}+\mathrm{H}_{2} \mathrm{O}^{+} \\
\mathrm{H}_{2} \mathrm{O}^{+}+\mathrm{H}_{2} \mathrm{O} \rightarrow \mathrm{H}_{3} \mathrm{O}^{+}+\mathrm{OH}
\end{gathered}
$$

\section{B. Polarity Effect}

\section{1) Power and Charge Transfer Comparison}

Table 2 shows a comparison of the power and charge transfer in air, when positive or negative polarities was applied. The average charge transfer per pulse was 57.5 $\mathrm{nC}$ for positive polarity, and $21 \mathrm{nC}$ for negative polarity, corresponding to $115 \mu \mathrm{C} / \mathrm{s}$ for positive polarity and $64 \mu \mathrm{C} / \mathrm{s}$ for negative polarity, respectively. The input power and charge transfer in positive air streamer discharges are roughly twice that for negative air streamer discharges.

Table 2. Power and charge transfer for positive and negative discharges in air.

\begin{tabular}{|c|c|c|c|}
\hline Polarity & $\begin{array}{c}\text { Power } \\
(\mathrm{W})\end{array}$ & $\begin{array}{c}\text { Charge } \\
\text { transfer/pulse } \\
(\mathrm{nC})\end{array}$ & $\begin{array}{c}\text { Charge } \\
\text { Transfer/s } \\
(\mathrm{uQ})\end{array}$ \\
\hline Negative & 0.063 & 21 & 64 \\
\hline Positive & 0.125 & 57.5 & 115 \\
\hline
\end{tabular}

\section{2) Hydroxyl Radical Production}

Polarity effects in air were compared. The number of hydroxyl radicals produced under both polarities increased linearly with time. For 15 minutes treatment, the hydroxyl radical production rates were similar, being $2.7 \times 10^{-7} \mathrm{Ms}^{-1}$ for positive air discharges, and $2.6 \times 10^{-7} \mathrm{Ms}^{-1}$ for negative air discharges.

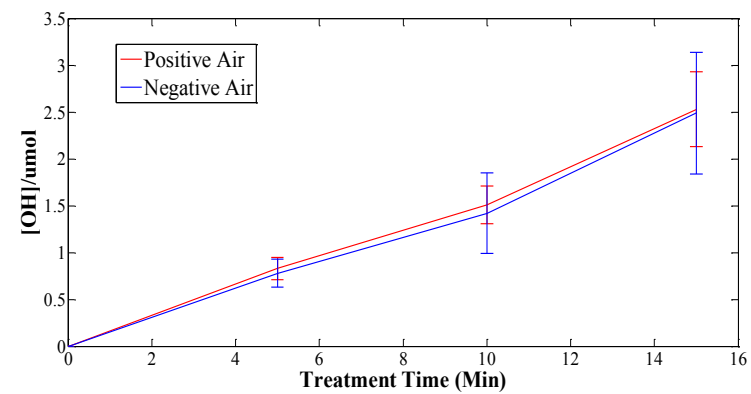

Figure 5. Hydroxyl radical production in air for both polarities.

In negative streamer discharges, energetic electrons are considered to be the main contribution to hydroxyl production. Electrons can attach with oxygen molecules to form oxygen atoms (Eq. (11) and Eq. (12)), which can react with water to produce hydroxyl radicals. Electrons can also react with water molecules directly to form hydroxyl radicals (Eq. (13) to Eq. (15)). The hydrogen atom from the reaction detailed in Eq. (14) 
can further react, directly or indirectly, with superoxide to form hydroxyl radicals (Eq. (16) to (Eq. (18)).

Electron attachment reactions:

Electron reactions:

$$
\begin{gathered}
\mathrm{O}_{2}+\mathrm{e} \rightarrow \mathrm{O}+\mathrm{O}^{-}(\mathrm{E}=6.7 \mathrm{eV}) \\
\mathrm{O}+\mathrm{H}_{2} \mathrm{O} \rightarrow \mathrm{OH}+\mathrm{OH}
\end{gathered}
$$

$$
\begin{aligned}
& \mathrm{H}_{2} \mathrm{O}+\mathrm{e} \rightarrow \mathrm{H}^{-}+\mathrm{OH}(\mathrm{E}=6.5 \mathrm{eV}) \\
& \mathrm{H}_{2} \mathrm{O}+\mathrm{e} \rightarrow \mathrm{H}+\mathrm{OH}(\mathrm{E}=4.8 \mathrm{eV})
\end{aligned}
$$

$2 \mathrm{H}_{2} \mathrm{O}+\mathrm{e} \rightarrow \mathrm{H}_{3} \mathrm{O}^{+}+\mathrm{OH}+2 \mathrm{e}(\mathrm{E}=12.6 \mathrm{eV})$

Hydrogen atom reactions:

$$
\begin{gathered}
\mathrm{H}+\mathrm{O}_{2} \rightarrow \mathrm{HO}_{2} \\
\mathrm{O}+\mathrm{HO}_{2} \rightarrow \mathrm{OH}+\mathrm{O}_{2} \\
\mathrm{H}+\mathrm{HO}_{2} \rightarrow 2 \mathrm{OH}
\end{gathered}
$$

\section{Energy Efficiency of Hydroxyl Radical Production}

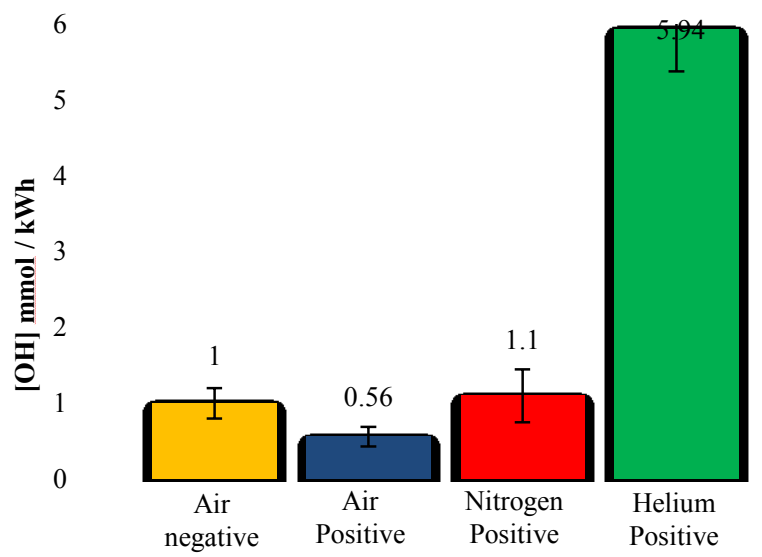

Figure 6. Hydroxyl radical production energy efficiency.

The energy efficiency for hydroxyl radical production in air is $1 \mathrm{mmol} / \mathrm{kWh}$ for negative discharges and $0.56 \mathrm{mmol} / \mathrm{kWh}$ for positive discharges. Although the power and charge transfer per second for positive discharges are almost twice as high than for negative discharges, negative polarity yields $80 \%$ higher energy efficiency than positive polarity for hydroxyl production using air.

In positive streamer discharge, helium has the highest energy efficiency which is $5.94 \mathrm{mmol} / \mathrm{kWh}$, compared to $1.1 \mathrm{mmol} / \mathrm{kWh}$ using nitrogen and $0.56 \mathrm{mmol} / \mathrm{kWh}$ using air. Hydroxyl radical production is most efficient using helium, as the power and charge transfer per second are $108 \%$ and $82 \%$ higher, respectively, than using air, and $44 \%$ and $22 \%$ higher, respectively, than using nitrogen. The energy efficiency for hydroxyl radical production using helium is 5.4 times higher than for nitrogen, and 10.6 times higher than for air.

\section{CONCLUSION}

The positive discharge in helium demonstrated higher power consumption and charge transfer per second and higher hydroxyl radical production energy efficiency of $5.94 \mathrm{mmol} / \mathrm{kWh}$, compared to $1.1 \mathrm{mmol} / \mathrm{kWh}$ using nitrogen and $0.56 \mathrm{mmol} / \mathrm{kWh}$ using air. This may be explained by the high peak current of $\sim 750 \mathrm{~mA}$ measured in Helium compared to the $\sim 50 \mathrm{~mA}$ in air and $\sim 80 \mathrm{~mA}$ in nitrogen. The helium positive ions in the streamer also move faster to the water surface compared with oxygen and nitrogen ions.

Negative streamer discharges in air have $80 \%$ higher energy efficiency than that positive streamer discharges in air, although the power and charge transfer per second are only $50 \%$ and $56 \%$ of that for positive discharges, respectively. The chemical reactions to produce hydroxyl radicals in negative discharges are more complicated than for positive discharge, as electron attachment reactions, direct electron reactions and hydrogen atom reactions all contribute, rather than only ionization reactions, as in positive discharges.

The hydroxyl radical production rate was $2.6 \times$ $10^{-7} \mathrm{Ms}^{-1}$ in negative air discharges, and $2.7 \times$ $10^{-7} \mathrm{Ms}^{-1}, 1.8 \times 10^{-6} \mathrm{Ms}^{-1}, 2.2 \times 10^{-6} \mathrm{Ms}^{-1}$ in positive air, nitrogen and helium discharges, respectively.

\section{REFERENCE}

[1] O. Lesage and L. Falk, "Treatment of 4chlorobenzoic acid by plasma-based advanced oxidation processes", Chemical Engineering and Processing: Process Intensification., vol. 72, pp. 82-89, Oct. 2013

[2] R.W. Matthews, "The radiation chemistry of aqueous sodium terephthalate solutions", Australian atomic energy commission research establishment, Lucas heights, 1980.

[3] Y.S. Mok and J.O. Jo, "Application of dielectric barrier discharge reactor immersed in wastewater to the oxidative degradation of organic contaminant", Plasma Chem. Plasma Process., vol. 27, pp. 51-64, 2007

[4] Kristin Kutschera and Hilmar Bo", "Photoinitiated oxidation of geosmin and 2methylisoborneol by irradiation with $254 \mathrm{~nm}$ and 185 nm UV light", Water Research., vol. 43, pp. 2224 2232, 2009

[5] L. O. de B. Benetolia and B. M. Cadorina, "Pyrite-enhanced methylene blue degradation in nonthermal plasma water treatment reactor", Journal of Hazardous Materials., vol. 237- 238, pp. 55- 62, Oct. 2012

[6] Kanazawa S and Kawano H, "Observation of $\mathrm{OH}$ radicals produced by pulsed discharges on the surface of a liquid", Plasma Sources Sci. Technol., vol. 20, no. 3, 2011

[7] Kanazawa S and Furuki T, "Measurement of $\mathrm{OH}$ Radicals in Aqueous Solution Produced by Atmospheric-pressure LF Plasma Jet", Electrostatics Society of America., vol. N, no. 3, 2012

[8] Li S, Hu S and Zhang H, "Formation of Hydroxyl Radicals and Hydrogen Peroxide by a Novel Nanosecond Pulsed Plasma Power in Water", 2012 IEEE Trans. Plasma Sci., vol. 40, pp. 63-67, 2012 